\title{
Trasplante autólogo de precursores hematopoyéticos en pacientes mayores de 65 años, experiencia en un centro médico de Cali (Colombia)
}

\author{
Autologous transplantation of hematopoietic precursors in patients over 65 years, \\ experience in a medical center in Cali (Colombia)
}

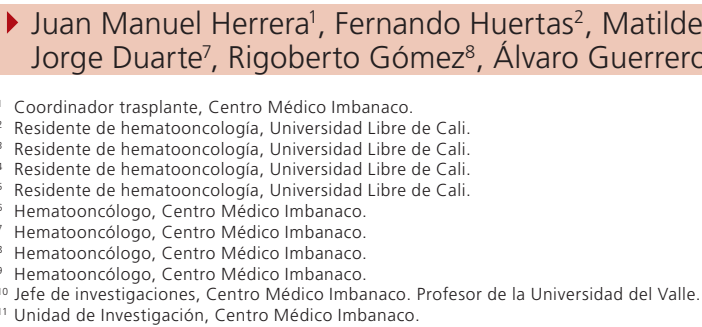

\section{Resumen}

El trasplante de células hematopoyéticas $(\mathrm{TCH})$ tiene como objetivo proporcionar al receptor un sistema hematopoyético nuevo que funcione permanentemente, con el objetivo de curar enfermedades hematológicas benignas y neoplásicas, así como algunos tumores sólidos, en diferentes edades; sin embargo, se considera que en edad avanzada, mayores de 65 años, es una contraindicación relativa.

Objetivo: describir las características clínicas y desenlaces de pacientes que recibieron trasplante de células hematopoyéticas mayores de 65 años en un centro de referencia en Cali.

Materiales y métodos: estudio descriptivo retrospectivo basado en los registros médicos de toda la población con TCH autólogo durante el período 2010 a 2014. Se tuvieron en cuenta el sexo, la edad, las comorbilidades, el índice de Sorror, la recuperación hematológica y la mortalidad al día 100.

Resultados: se trasplantaron 13 pacientes. El 54\% (7) eran hombres. El 70\% tenía diagnóstico de mieloma múltiple; el 30\% (4), HTA. El 97\% de los pacientes presentó un índice de Sorror bajo e intermedio, no hubo mortalidad al día 100, 2 pacientes fallecieron en recaída posteriormente. La mediana de seguimiento fue 21 meses. La complicación más común asociada a trasplante fue mucositis, seguida por neutropenia febril.

Conclusiones: el trasplante de médula ósea en pacientes mayores de 65 años es una opción de tratamiento seguro, la edad no debe ser un obstáculo para su realización, pues se obtiene una buena tasa de respuesta con una mortalidad baja.

Palabras clave: trasplantes, enfermedades hematológicas, precursores hematopoyéticos.

\begin{abstract}
Hematopoietic cell transplantation (HCT) aims to provide the recipient with a new hematopoietic system that works permanently, aiming at curing benign and neoplastic hematological diseases, if, like some solid tumors, at different ages, it is considered that older, older 65 , is a relative contraindication.

Objective: To describe the clinical characteristics and outcomes of patients who received a hematopoietic cell transplant over 65 years of age at a reference center in Cali.

Materials and methods: A retrospective descriptive study based on the medical records of the entire autologous HCT population, from 2008 to 2013. Gender, age, comorbidities, Sorror index, hematologic recovery and mortality at day 100. Results: Thirteen patients were transplanted, 54\% (7) men, 70\% had a diagnosis of multiple myeloma, 30\% (4) had hypertension, $97 \%$ of the patients had a low and intermediate Sorror index, 100, 2 patients died in relapse later. The median
\end{abstract}

Recibido: 10 de octubre de 2016; aceptado: 25 de junio de 2018 
TRASPLANTE AUTÓLOGO DE PRECURSORES HEMATOPOYÉTICOS EN PACIENTES MAYORES DE 65 AÑOS, EXPERIENCIA EN UN CENTRO MÉDICO DE CALI (COLOMBIA)

follow-up was 21 months. The most common complication associated with transplantation was mucositis, followed by febrile neutropenia.

Conclusions: Bone marrow transplantation in patients over 65 years of age is an option for safe treatment; age should not be a limitation for its performance, since a good response rate with a low mortality is obtained.

Key words: Hematological diseases, hematopoietic stem cell transplantation, transplantation, autologous.

\section{Introducción}

El trasplante de células hematopoyéticas (TCH) es un término general que abarca una serie de procedimientos en los que el paciente es tratado con quimioterapia, radioterapia o ambos, seguido por la infusión de células madre hematopoyéticas/células progenitoras, y puede dividirse en subtipos basados en el tipo de donante, la fuente de células progenitoras hematopoyéticas y el régimen de preparación. Cada uno de estos factores influye en la eficacia y las toxicidades a largo plazo asociadas con el procedimiento'.

El trasplante tiene dos objetivos posibles: sustituir la hematopoyesis del paciente por ser total o parcialmente defectuosa, insuficiente o neoplásica; y permitir un tratamiento antineoplásico con dosis muy elevadas que origina mielosupresión prolongada o definitiva'.

El TCH es una opción de tratamiento importante y potencialmente curativa para una amplia variedad de enfermedades malignas y no malignas; es así como, según la fuente de progenitores hematopoyéticos, estos pueden ser de médula ósea, sangre periférica o cordón umbilical; $y$, de acuerdo con el tipo de trasplante, este puede ser alogénico o autólogo².

En los últimos años se han logrado significativas tasas de respuesta, hasta del $80 \%$ al $90 \%$, que dependen de la patología de base y del estado de la enfermedad, al igual que de la fuente de progenitores hematopoyéticos, el tipo de trasplante, la edad y las comorbilidades del receptor; sin embargo, hoy en día aún observamos complicaciones que pueden poner en peligro la vida del paciente o deteriorar su calidad de vida ${ }^{3}$.

En Colombia no hay reportes y la información que proporcione este estudio será un primer paso para comprender que la edad no es un factor excluyente para la realización del trasplante autólogo de progenitores hematopoyéticos.

Para categorizar a los pacientes mayores de 65 años conforme con sus comorbilidades, se utiliza una escala denominada índice de comorbilidad de Sorror ${ }^{4,5}$; en un estudio, el Dr. M. Sorror y sus colegas encontraron que las tasas a cinco años de la supervivencia global y libre de progresión de la enfermedad entre los pacientes con trasplantes autólogos fueron del 35\% y 32\%, respectivamente. Los pacientes se dividieron en tres grupos de edad: 60 a 64, 65 a 69 y de 70 a 75, con tasas de supervivencia comparables, lo que sugiere que la edad juega un papel limitado en la forma en que los pacientes toleran el trasplante. El aumento de los problemas médicos no relacionados con el cáncer (comorbilidades) y un mayor grado de agresividad del cáncer fueron los dos factores que afectaron la supervivencia entre los pacientes de mayor edad.

\section{Métodos}

Se efectuó un estudio retrospectivo basado en la revisión de registros médicos de todos los pacientes de 65 años o más llevados a trasplante autólogo de precursores hematopoyéticos entre el año 2010 y 2014. Se hizo un análisis descriptivo de las características generales de los pacientes. Se estimó la probabilidad de recuperación hematológica por medio del análisis de Kaplan-Meier. Todos los análisis fueron llevados a cabo en Stata 13 (StataCorp, College Station, TX).

\section{Resultados}

\section{Descripción general de los pacientes}

Se incluyeron 13 pacientes a los cuales se les realizó trasplante de células hematopoyéticas, el rango de edad fue de 65 a 73 años y el 46,1\% fueron mujeres. El diagnóstico más frecuente fue el mieloma múltiple y las comorbilidades de mayor prevalencia fueron la hipertensión arterial y la tuberculosis latente; otras de las comorbilidades presentes entre los pacientes fueron hepatitis, fibrilación auricular y osteoporosis. Por medio del índice de Sorror, se evidenció que la mayoría de pacientes fueron clasificados como riesgo bajo. El promedio de células progenitoras trasfundidas fue de 4,6 (tabla 1)

La mitad de los pacientes alcanzaron la recuperación hematológica antes del día +12 postrasplante; la primera recuperación ocurrió en el día 9 y la última, en el día 15 (figura 1). 
Tabla 1. Características generales de los pacientes sometidos a trasplante autólogo de precursores hematopoyéticos

\begin{tabular}{|c|c|}
\hline Características & $N=13$ \\
\hline \multicolumn{2}{|c|}{ Edad } \\
\hline $\begin{array}{l}\text { Media } \pm \text { SD } \\
\text { Mediana (rango) }\end{array}$ & $\begin{array}{l}67,8 \pm 2,8 \\
67(65-73)\end{array}$ \\
\hline \multicolumn{2}{|c|}{ Sexon (\%) } \\
\hline $\mathrm{F}$ & $6(46,1)$ \\
\hline \multicolumn{2}{|c|}{ Diagnóstico n (\%) } \\
\hline $\begin{array}{l}\text { Mieloma múltiple } \\
\text { Linfoma Hodgkin } \\
\text { Linfoma no Hodgkin } \\
\text { Linfoma del manto } \\
\text { Macroglobulinemia de Waldenström }\end{array}$ & $\begin{array}{l}9(69,2) \\
1(7,7) \\
1(7,7) \\
1(7,7) \\
1(7,7)\end{array}$ \\
\hline \multicolumn{2}{|c|}{ Comorbilidades $n(\%)$} \\
\hline $\begin{array}{l}\text { HTA } \\
\text { Hipotiroidismo } \\
\text { TBC latente } \\
\text { Otras }\end{array}$ & $\begin{array}{l}4(30,8) \\
3(23,1) \\
4(30,8) \\
5(38,5)\end{array}$ \\
\hline \multicolumn{2}{|c|}{ Índice de Sorror $n$ (\%) } \\
\hline $\begin{array}{l}\text { Bajo } \\
\text { Intermedio } \\
\text { Alto }\end{array}$ & $\begin{array}{l}8(61,5) \\
4(30,8) \\
1(7,7)\end{array}$ \\
\hline \multicolumn{2}{|c|}{$C D 34+10 \wedge 6$} \\
\hline $\begin{array}{l}\text { Media } \pm \text { SD } \\
\text { Mediana (rango) }\end{array}$ & $\begin{array}{c}4,6 \pm 1,7 \\
4,7(1,8-7,7)\end{array}$ \\
\hline
\end{tabular}

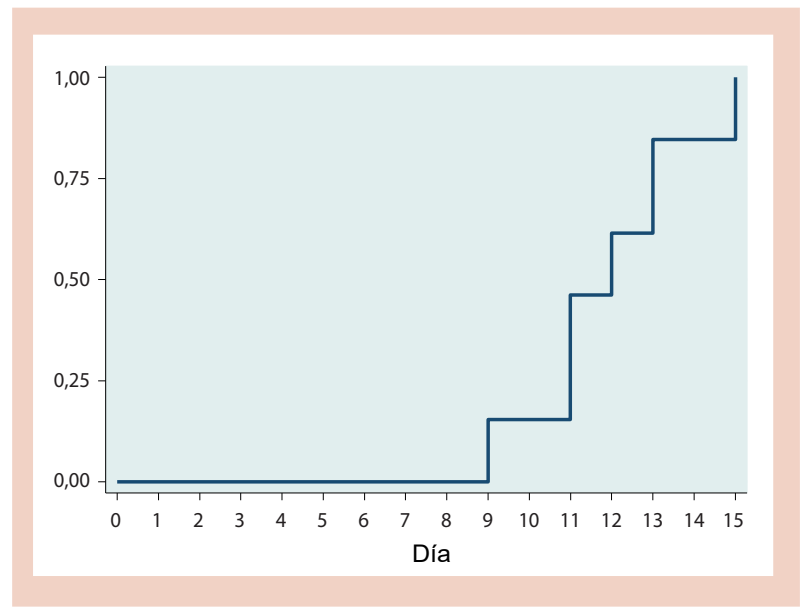

Figura 1. Probabilidad de recuperación hematológica.

La complicación más frecuente relacionada con el trasplante fue mucositis en la mitad de los pacientes, seguida por neutropenia febril. Otras complicaciones asociadas fueron hematuria, absceso perirrectal y colitis. De los pacientes trasplantados, 4 tuvieron recaída y, de estos, 2 fallecieron al mes 29 y 50 postrasplante (muertes no vinculadas con el trasplante). Nueve pacientes alcanzaron la remisión con un promedio de seguimiento de 21 meses (tabla 2).

\section{Discusión}

La incorporación de puntos de corte de edad en los criterios de elegibilidad para el trasplante
Tabla 2. Resultados clínicos de los pacientes sometidos a trasplante autólogo de precursores hematopoyéticos $(\mathrm{N}=13)$

\begin{tabular}{|l|c|}
\hline \multicolumn{1}{|c|}{ Características complicaciones } & - $(\%)$ \\
\hline Mucositis & $7(53,8)$ \\
Septicemia & $2(15,4)$ \\
Infección urinaria & $2(15,4)$ \\
Neutropenia febril & $3(23,1)$ \\
Otra & $5(38,5)$ \\
\hline \multicolumn{2}{|c|}{ Mortalidad día 100 } \\
\hline Sí Estado actual \\
\hline \multicolumn{2}{|c|}{$0(0,0)$} \\
\hline Fallece & $2(15,4)$ \\
Recaída & $2(15,4)$ \\
Remisión & $9(69,2)$ \\
\hline
\end{tabular}

autólogo de células hematopoyéticas es controvertido ${ }^{6,7}$. Los estudios iniciales que demuestran el beneficio de trasplante en diversas patologías se han realizado en pacientes más jóvenes ( $<65$ años); sin embargo, existen algunos reportes que sugieren que el beneficio de trasplante autólogo se puede observar en los adultos mayores ${ }^{8}$. En muchos países europeos, el trasplante autólogo se ofrece principalmente a pacientes menores de 65 años de edad ${ }^{8}$. En los Estados Unidos, no se utiliza un límite de edad estricta, las decisiones se toman dependiendo de cada caso según la patología y las comorbilidades de los pacientes 9 .

Este es el primer estudio reportado en Colombia y es el primer paso para comprender que la edad no es un factor excluyente para la realización del trasplante autólogo de progenitores hematopoyéticos, así como cuáles pueden ser las mejores opciones de regímenes de condicionamiento y los resultados.

La mayoría de los datos sobre el impacto del trasplante autólogo de células hematopoyéticas proviene de estudios en pacientes con mieloma múltiple ${ }^{10-13}$, dada la alta prevalencia de dicha enfermedad en este grupo etario. En nuestro estudio, el $69 \%$ de los pacientes tenía este diagnóstico al momento de ingresar al protocolo de trasplante, lo cual se encuentra en concordancia con lo reportado mundialmente ${ }^{14}$.

Existen menos reportes acerca de resultados de pacientes mayores sometidos a trasplante autólogo de precursores hematopoyéticos para neoplasias malignas hematológicas distintas de mieloma. Elstrom et al. efectuaron un estudio retrospectivo de pacientes con linfomas mayores de 69 años llevados a trasplante en el Weill Cornell Medical College, en el que se reportaron 21 pacientes con una edad mediana de 71 años (rango 69-86). El 76\% de estos pacientes logró respuesta completa luego 
del trasplante. Con una mediana de seguimiento de 20 meses, la supervivencia libre de progresión y la sobrevida global media eran de 19 y 18 meses, respectivamente. La mortalidad relacionada con el trasplante al día 100 fue del $9 \%{ }^{15}$. En nuestro estudio, tres pacientes con linfomas cumplieron los criterios de inclusión. La complicación más frecuente asociada al trasplante fue mucositis en la mitad de los pacientes, seguida por neutropenia febril; encontramos un $0 \%$ de mortalidad relacionada con el día 100.

El European Group for Blood and Marrow Transplantation (EBMT) analizó los datos de 712 pacientes con linfoma de células; la mediana de tiempo desde el diagnóstico hasta el trasplante fue mayor en los pacientes de edad avanzada (11 meses frente a 9, $p=0,005)$; la mediana de seguimiento después del trasplante fue de 19 y 25 meses, respectivamente. No hubo diferencias en la tasa de recaída (66\% versus $55 \%$ a 5 años), PFS (29\% versus $40 \%$ ) y OS $(61 \%$ versus $67 \%$ ) entre ambas poblaciones de pacientes.
Este grupo concluye que el trasplante más allá de 65 años de edad es factible en pacientes seleccionados con linfoma de células de manto, y los resultados en el control de enfermedades y la supervivencia son similares a los de pacientes más jóvenes ${ }^{16}$. Dentro de los pacientes estudiados en nuestro centro, solo un paciente con linfoma de células del manto fue incluido.

La edad biológica no debe ser el único criterio usado para determinar la elegibilidad de los pacientes que requieren ser llevados a un trasplante autólogo de precursores hematopoyéticos; sin embargo, puede indicar la necesidad de ajustar la dosis del régimen de acondicionamiento.

\section{Conclusión}

El trasplante de médula ósea en pacientes mayores de 65 años es una opción de tratamiento seguro, la edad no debe ser un obstáculo para su realización, pues se obtiene una buena tasa de respuesta con una mortalidad baja.

\section{Referencias}

1. Hatzimichael $\mathrm{E}$, Tuthill M. Hematopoietic stem cell transplantation. Stem Cells Cloning. 2010;3:105-17.

2. Majhail NS, Farnia SH, Carpenter PA, Champlin RE, Crawford S, Marks DI, et al. Indications for autologous and allogeneic hematopoietic cell transplantation: guidelines from the American Society for Blood and Marrow Transplantation. Biol Blood Marrow Transplant [internet]. 2015;21(11):1863-9. Disponible en: http://dx.doi. org/10.1016/j.bbmt.2015.07.032

3. Khaled SK, Thomas SH, Forman SJ. Allogeneic hematopoietic cell transplantation for acute lymphoblastic leukemia in adults. Curr Opin Oncol. 2012;24(2):182-90.

4. Sorror ML. How I assess comorbidities before hematopoietic cell transplantation. Blood. 2013;121(15):2854-63.

5. Sorror ML, Maris MB, Storb R, Baron F, Sandmaier BM, Maloney DG, et al. Hematopoietic cell transplantation (HCT)-specific comorbidity index: a new tool for risk assessment before allogeneic HCT. 2005;106(8):2912-9.

6. Mohty M, Harousseau JL. Treatment of autologous stem cell transplant-eligible multiple myeloma patients: ten questions and answers. Haematologica [internet]. 2014;99(3):408-16. Disponible en: http://www.haematologica.org/cgi/doi/10.3324/ haematol.2013.096149

7. Auner HW, Garderet L, Kröger N. Autologous haematopoietic cell transplantation in elderly patients with multiple myeloma. Br J Haematol. 2015;171(4):453-62.

8. Brenner $\mathrm{H}$, Gondos $A$, Pulte $D$. Recent major improvement in long-term survival of younger patients with multiple myeloma. Blood. 2008;111(5):2521-6.
9. Majhail NS, Farnia SH, Carpenter PA, Champlin RE, Crawford $S$, Marks DI, et al. Indications for autologous and allogeneic hematopoietic cell transplantation: guidelines from the American Society for Blood and Marrow Transplantation. Biol Blood Marrow Transplant [internet]. 2015;21(11):1863-9. Disponible en: https://www.ncbi.nlm.nih.gov/pmc/articles/PMC4830270/ pdf/nihms-770692.pdf

10. Thanendrarajan S, Stein CK, Davies FE, Van Rhee F, Zangari $M$, Heuck $C$, et al. Defining the impact of tandem autologous stem cell transplantation in multiple myeloma: a casematch analysis in the total therapy trials. Blood [internet]. 2015;126(23):3182. Disponible en: http://www.bloodjournal. org/content/126/23/3182.abstract

11. Balsam L, Saad C, Arsene C, Fogel J. Impact of autologous stem cell transplantation on blood pressure and renal function in multiple myeloma patients. J Natl Med Assoc [internet]. 2017;109(3):182-91. Disponible en: http://dx.doi. org/10.1016/j.jnma.2017.02.010

12. Alyea E, Weller E, Schlossman R, Canning C, Mauch $P$, $\mathrm{Ng} \mathrm{A}$, et al. Outcome after autologous and allogeneic stem cell transplantation for patients with multiple myeloma: impact of graft-versus-myeloma effect. Bone Marrow Transplant [internet]. 2003;32(12):1145-51. Disponible en: http://www.nature.com/doifinder/10.1038/ sj.bmt. 1704289

13. Kumar L, Gogi R, Patel AK, Mookerjee A, Sahoo RK, Malik PS, et al. Multiple myeloma with extramedullary disease: impact of autologous stem cell transplantation on outcome. Bone Marrow Transplant [internet]. 2017;52(10):1473-5. Disponible en: http://dx.doi.org/10.1038/bmt.2017.165 
14. McCarthy PL Jr, Hahn T, Hassebroek A, Bredeson C, Gajewski J, Hale $G$, et al. Trends in use of and survival after autologous hematopoietic cell transplantation in North America, 1995-2005: significant improvement in survival for lymphoma and myeloma during a period of increasing recipient age. Biol Blood Marrow Transplant. 2013;19(7):1116-23.

15. Elstrom R, Martin P, Ostrow K, Barrientos J, Chadburn A, Furman $R$, et al. Response to second-line therapy defines the potential for cure in patients with recurrent diffuse large B-cell lymphoma: implications for the development of novel therapeutic strategies. Clin Lymphoma Myeloma Leuk. 2010;10(3):192-6.

16. Jantunen E, Lemoli R. Preemptive use of plerixafor in difficult-to-mobilize patients: an emerging concept. Transfusion. 2012;52(4):906-14. 